\title{
Effect of Ectopic Beats on Heart Rate Variability Indices in Congestive Heart Failure Patients
}

\author{
Chengyu Liu ${ }^{1}$, Lina Zhao ${ }^{1,2}$, Zhipeng Cai ${ }^{1}$, Feifei Liu ${ }^{1}$, Shoushui Wei ${ }^{2}$, Jianqing Li ${ }^{1,3}$ and Alan Murray ${ }^{4}$ \\ ${ }^{1}$ School of Instrument Science and Engineering, Southeast University, Nanjing, China \\ ${ }^{2}$ School of Control Science and Engineering, Shandong University, Jinan, China \\ ${ }^{3}$ School of Basic Medical Sciences, Nanjing Medical University, Nanjing, China \\ ${ }^{4}$ School of Engineering, Newcastle University, Newcastle upon Tyne, UK
}

\begin{abstract}
Heart rate variability (HRV) provides an important insight for understanding the underlying mechanisms of the cardiovascular system, and provides a valuable tool for early detection of cardiovascular abnormalities. Ectopic beats have been proven to have an influence on HRV results, but the effect of different amounts of ectopic beats on analysis of congestive heart failure (CHF) patient rhythms has not been quantified. In this study, we tested the commonly used HRV indices for significant differences between 5-min RR segments with and without ectopic beats. Eight long-term CHF RR interval recordings from http://www.physionet.org were studied. Each recording (about $24 \mathrm{~h}$ ) was divided into non-overlapping segments of 5-min RR segments without or with different numbers of ectopic beats. Two time-domain HRV indices of SDNN and RMSSD and two frequency-domain indices of normalized low frequency (LFn) and high frequency (HFn) powers were employed. Results showed that ectopic segments had significantly larger values for SDNN (39 \pm 18 vs. ectopic free segments $28 \pm 16 \mathrm{~ms}, P<0.05)$, RMSSD (47 \pm 29 vs. $24 \pm 23 \mathrm{~ms}, P<0.05)$ and HFn $(0.66 \pm 0.13$ vs. $0.52 \pm 0.14, P<0.01$, and significantly lower values for $\mathrm{LFn}$ $(0.34 \pm 0.13$ vs. ectopic free segments $0.48 \pm 0.14, P<0.01)$. Compared with the indices of RMSSD and frequency-domain indices, SDNN was least affected by a relatively small amount of ectopic beats (one to six beats). Compared with the time-domain indices, the frequency-domain indices responded more quickly to the appearance of ectopic beats. The results confirmed that ectopic beats in RR segments have a significant influence on the HRV results for CHF patients.
\end{abstract}

Keywords - Electrocardiographic (ECG), Heart rate variability (HRV), Ectopic beat, Congestive heart failure (CHF).

\section{INTRODUCTION}

Heart rate variability (HRV) studies the slight fluctuations in RR interval time series and has become an important tool for the assessment of cardiovascular autonomic regulation during various physiological and clinical conditions. However, ectopic beats in RR interval time series may cause bias in the reliable measurement of HRV [1, 2, 3]. Researchers reported that some HRV time-domain parameters are more sen- sitive to ectopic beats than others [2]. Researchers also stated that the presence of only one ectopic beat can introduce an increase in the high frequency (HF) power in HRV of around $10 \%$ [4]. Non-linear index of HRV was also influenced by ectopic beats [5]. The reason is that ectopic beats can induce the sudden changes in RR intervals and increase the high frequency fluctuation in the tachogram of HRV, distorting the true measure of an HRV metric [6].

Although many detecting and editing (correcting) methods for ectopic beats have been proposed [3, 7, 8, 9], there is no agreed conclusion on which method is more efficient to handle the ectopic beats to obtain accurate HRV estimation $[3,6,9]$. Meanwhile, the effect of different amounts of ectopic beats on the common time-domain and frequencydomain HRV indices for special cardiovascular disease has not been widely documented. This paper, therefore, aims to quantify the differences between ectopic-free and ectopic RR interval time series for congestive heart failure (CHF) patients, and to quantify the effect of different amounts of ectopic beats on the commonly used time-domain and frequency-domain HRV indices.

\section{METHODS}

\section{A. Database}

All data used were from the PhysioNet CHF RR Interval Databases in http://www.physionet.org [10], a free-access, on-line archive of physiological signals. This database included 29 long-term RR interval records of subjects aged 34 to 79, with CHF (NYHA classes I, II, and III). The original ECG signals were digitized at $128 \mathrm{~Hz}$, and the beat annotations were obtained by automated analysis with manual review and correction.

A 5-min time window was used to segment the long-term RR interval records. In each 5-min RR segment, RR intervals greater than $2 \mathrm{~s}$ were firstly removed to exclude the influence from artifacts [11]. Then 5-min RR segments were classified as an ectopic segment if at least one ectopic beat was included 
or ectopic-free segment if no ectopic beat existed. Ectopic beats were from supra-ventricular or ventricular beats, depending on the localization of ectopic focus. Figure 1 shows the examples of an ectopic-free 5-min RR segment (upper panel) and an ectopic 5-min RR segment (lower panel). Table 1 summarizes the numbers of normal and ectopic beats in each of the 29 records, as well as the numbers of ectopicfree and ectopic 5-min RR segments. To exclude the record effect, only eight records with approximately equal amounts of ectopic-free and ectopic RR segments were selected from the 29 records for the following analysis, which were marked in bold in Table 1 .


Fig. 1: Examples of 5-min RR segments: (A) ectopic-free RR segment and (B) RR segment with A (atrial) type ectopic beat.

\section{B. HRV indices}

For a 5-min RR segment $x(i),(i=1,2,, N)$, four common HRV indices were calculated in this study. 1) Time-domain indices The standard deviation of 5-min RR segment (SDNN) and the square root of the mean of the sum of the squares of differences between adjacent intervals of 5-min RR segment (RMSSD) were used and were defined as:

$$
\begin{gathered}
S D N N=\sqrt{\frac{1}{N-1} \sum_{i=1}^{N}(x(i)-\bar{x})^{2}} \\
R M S S D=\sqrt{\frac{1}{N-1} \sum_{i=1}^{N-1}(x(i+1)-x(i))^{2}}
\end{gathered}
$$

where $\bar{x}=\frac{1}{N} \sum_{i=1}^{N} x(i)^{2}$ is the mean value of RR intervals.

2) Frequency-domain indices Frequency-domain analysis provides a quantitative evaluation of sympathetic and parasympathetic activation. The low frequency (LF) component (between $0.04-0.15 \mathrm{~Hz}$ ) reflects both sympathetic and
Table 1: Data profile for the PhysioNet CHF RR Interval Databases. Records in bold means the selected records with approximately equal

\begin{tabular}{|c|c|c|c|c|}
\hline \multirow[t]{2}{*}{ Record } & \multicolumn{2}{|l|}{ \# beats } & \multicolumn{2}{|c|}{ \# RR segments } \\
\hline & Normal & Ectopic & Ectopic-free & Ectopic \\
\hline chf201 & 112,119 & 61 & 240 & 36 \\
\hline $\operatorname{chf} 202$ & 109,003 & 273 & 97 & 150 \\
\hline $\operatorname{chf} 203$ & 98,473 & 496 & 75 & 187 \\
\hline $\operatorname{chf} 204$ & 96,270 & 2,297 & 0 & 247 \\
\hline $\operatorname{chf} 205$ & 132,931 & 1,356 & 31 & 245 \\
\hline $\operatorname{chf} 206$ & 124,341 & 11,112 & 11 & 240 \\
\hline $\operatorname{chf} 207$ & 94,064 & 15,189 & 1 & 249 \\
\hline $\operatorname{chf} 208$ & 105,327 & 3,073 & 31 & 257 \\
\hline $\operatorname{chf} 209$ & 108,758 & 507 & 70 & 156 \\
\hline $\operatorname{chf} 210$ & 139,636 & 2,122 & 16 & 258 \\
\hline $\operatorname{chf} 211$ & 127,194 & 14 & 275 & 116 \\
\hline $\operatorname{chf} 212$ & 98,972 & 3,483 & 0 & 2055 \\
\hline $\operatorname{chf} 213$ & 93,961 & 10,968 & 7 & 281 \\
\hline $\operatorname{chf} 214$ & 72,718 & 21,160 & 0 & 204 \\
\hline $\operatorname{chf} 215$ & 141,898 & 5,851 & 110 & 166 \\
\hline $\operatorname{chf} 216$ & 101,670 & 18 & 250 & 14 \\
\hline $\operatorname{chf} 217$ & 105,544 & 779 & 53 & 228 \\
\hline $\operatorname{chf} 218$ & 102,097 & 2,667 & 47 & 217 \\
\hline $\operatorname{chf} 219$ & 90,475 & 37 & 236 & 28 \\
\hline $\operatorname{chf} 220$ & 136,929 & 820 & 138 & 143 \\
\hline $\operatorname{chf} 221$ & 100,139 & 11,608 & 0 & 276 \\
\hline $\operatorname{chf} 222$ & 98,844 & 2,796 & 1 & 274 \\
\hline $\operatorname{chf} 223$ & 107,377 & 5,410 & 0 & 274 \\
\hline $\operatorname{chf224}$ & 134,367 & 356 & 137 & 150 \\
\hline $\operatorname{chf} 225$ & 91,161 & 242 & 97 & 121 \\
\hline $\operatorname{chf} 226$ & 129,508 & 1,638 & 18 & 257 \\
\hline $\operatorname{chf} 227$ & 101,032 & 5,649 & 0 & 275 \\
\hline $\operatorname{chf} 228$ & 117,459 & 1,467 & 71 & 204 \\
\hline chf229 & 122,656 & 22 & 267 & 20 \\
\hline
\end{tabular}
amounts of ectopic-free and ectopic RR segments.

parasympathetic nervous systems while the HF component (between $0.15-0.40 \mathrm{~Hz}$ ) mainly reflects vagal activity [6, 12]. Burg modern spectrum estimation was used to acquire frequency parameters. The spectral powers of LF and HF were normalized using $L F n=\frac{L F}{L F+H F}$ and $H F n=\frac{H F}{L F+H F}$.

\section{Statistical analysis}

Normal distributions of HRV indices were tested by the Kolmogorov-Smirnov test. All tested indices obeyed normal distribution. Hence, a group t-student test was used to determine whether the results obtained from the ectopic-free and ectopic RR segments had significant differences. Comparisons were also performed between the ectopic-free group and the groups with differnet amound of ectopic beats. All 
statistical analyses were performed using the SPSS software (Ver. 20, IBM, USA). A statistical significance was accepted at $P<0.05$.

\section{RESULTS}

Table 2 gives the overall means and SDs of HRV indices (i.e., SDNN, RMSSD, LFn and HFn) from both ectopic-free and ectopic 5-min RR segments. As shown in Table 2, there were significant differences in all four indices between the two groups (all $P<0.05$ ). For time-domain indices, SDNN in ectopic group was significantly larger by $11 \mathrm{~ms}(39 \pm 18 \mathrm{vs}$ $28 \pm 16 \mathrm{~ms}, P<0.05)$ and RMSSD was significantly larger by $23 \mathrm{~ms}$ ( $47 \pm 29$ vs $24 \pm 23 \mathrm{~ms}, P<0.05$ ). For frequency-domain indices, LFn in ectopic group was significantly lower by 0.14 $(0.34 \pm 0.13$ vs $0.48 \pm 0.14, P<0.01)$ while $H F n$ was significantly larger by $0.14(0.66 \pm 0.13$ vs $0.52 \pm 0.14, P<0.01)$.

Table 2: HRV results for ectopic-free and ectopic 5-min RR segments from the eight RR records used.

\begin{tabular}{llll}
\hline Index & Ectopic-free & Ectopic & $P$-value \\
\hline \# segment & 795 & 1,277 & - \\
\hline SDNN (ms) & $28 \pm 16$ & $39 \pm 18$ & $<0.05$ \\
RMSSD (ms) & $24 \pm 23$ & $47 \pm 29$ & $<0.05$ \\
LFn & $0.48 \pm 0.14$ & $0.34 \pm 0.13$ & $<0.01$ \\
HFn & $0.52 \pm 0.14$ & $0.66 \pm 0.13$ & $<0.01$ \\
\hline
\end{tabular}

Then we divided the ectopic RR segments into sub-groups with differnet amounts of ectopic beats. Figure 2 shows the distribution of ectopic-free and ectopic RR segments classified by the number of ectopic beats. There are total 795 ectopic-free RR segments (also as shown in Table 2). For ectopic ones, there are 464, 243, 138, 81, 50 and 33 segments when ectopic beats are from one to six respectively, and there are 217 ectopic segments with ectopic beats more than six. Figure 3 shows the corresponding mean and SDs values of the four HRV indices for each RR segment group, to allow the ectopic beat number-related HRV changes to be observed and compared.

For time-domain indices, SDNN increased with increasing ectopic beat number. When compared with the ectopicfree group, statistically significant increases were observed at the group with one ectopic beat $(P<0.01)$, the group with two ectopic beats $(P<0.01)$ and the group with more than six ectopic beats $(P<0.01)$. However, the groups with three to six ectopic beats did not show the statistical significance. The reason maybe come from the small amount of RR segments. RMSSD also increased with the increase of ectopic beat number for each group. When compared with the ectopic-free group, statistically significant increases were observed for all seven ectopic groups (all $P<0.01$ ).

For frequency-domain indices, LFn gradually decreased with the increase of ectopic beat number for each group. When compared with the ectopic-free group, statistically significant decreases were observed for all seven ectopic groups (all $P<0.01$ ). HFn showed a reverse trend to LFn since mathematically, the sum of LFn and HFn was a constant. For comparison of the results from time-domain and frequecy-domain HRV indices, the latter responded more quickly to the appearance of ectopci beats. With the increase of ectopic beats from one to six, LFn (HFn) quickly decreased (increased) at these situations with relatively small amount of ectopic beats. When the number of ectopic beats was large and increased up to more than six, the decrease trend in LFn (increase trend in HFn) was not obvious. However, time-domian indices showed distinct changes when applied on the RR segments with more than six ectopic beats.

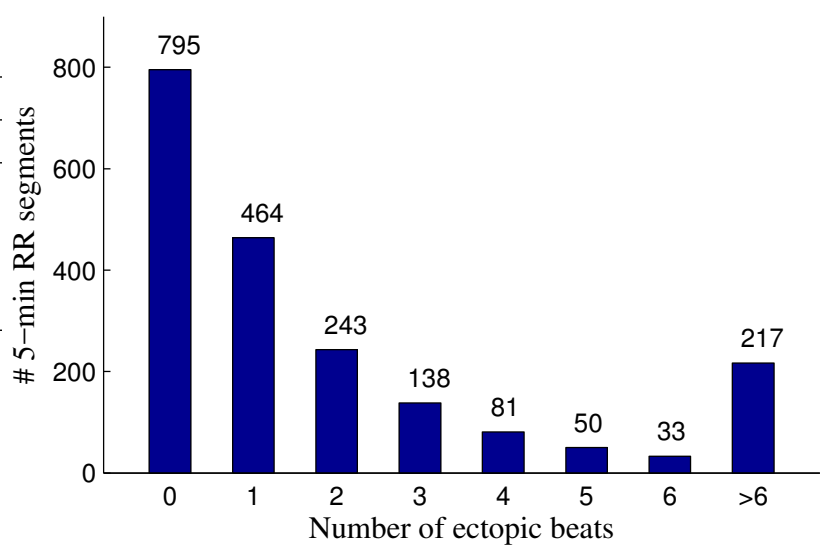

Fig. 2: Distribution of the ectopic-free and ectopic 5-min RR segments classified by the number of ectopic beats.

\section{DISCUSSION}

In this study, we quantified the effect of different amounts of ectopic beats on the common time-domain and frequencydomain HRV indices for CHF patients. The results showed that the ectopic RR segments had larger SDNN, RMSSD and HFn but lower LFn values than the ectopic-free RR segments. Compared with the indices of RMSSD and frequencydomain indices, SDNN was least affected by a relatively small amount of ectopic beats (one to six beats). Compared with the time-domain indices, the frequecy-domain indices responded more quickly to the appearance of ectopic beats. The results confirmed that ectopic beats in RR segments have 

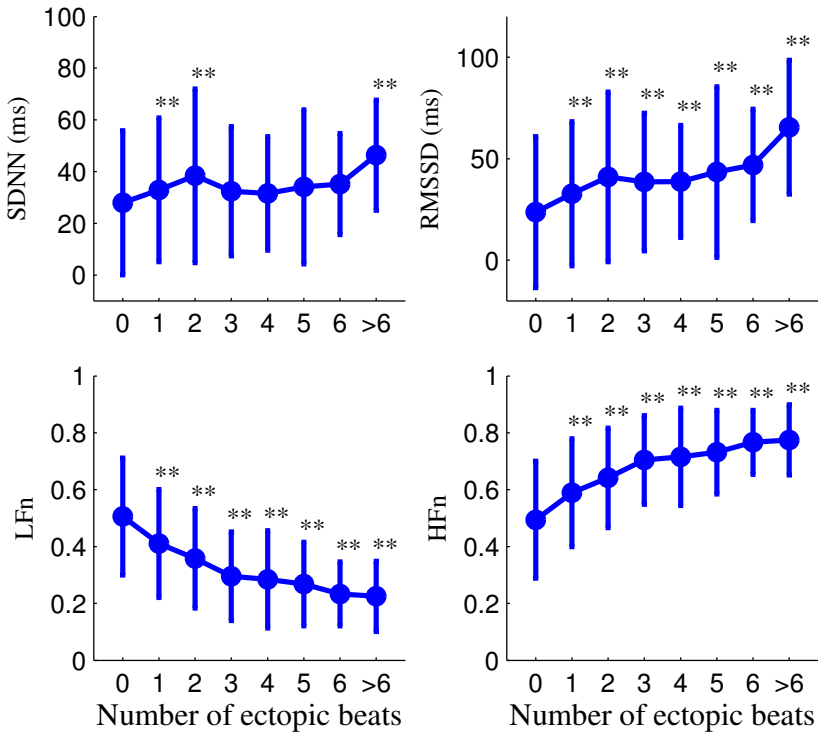

Fig. 3: Group mean and standard standards (SDs) of four HRV indices at each RR segment group with different numbers of ectopic beats. Compared with ectopic-free group, statistically significant differences at the $P<0.01$ level are marked as **.

a significant influence on the HRV results for CHF patients.

Ectopic beats are routinely removed (i.e., edited) from the RR tachogram prior to HRV analysis. Our results agreed with the previously reported works. Salo et al found that SDNN was least affected by the editing of RR intervals, and compared with the indices of SDNN and RMSSD, frequencydomain indices were more sensitive to the editing of RR intervals and reported larger errors after the ectopic beat editing [2]. This finding was consistent with our current study, where we showed that RMSSD and frequency-domain indices were more sensitive to the appearance of a small amount of ectopic beats (one to six beats). The reason is that the ectopic beats are usually step-like shapes and can result in sudden changes in RR interval time series. This effect is significant on the transient change of HRV reflected by RMSSD and the spectral components of HRV reflected by the frequency-domain LFn and HFn indices [6].

\section{CONFLICT OF INTEREST}

The authors declare no conflict of interest.

\section{ACKNOWLEDGEMENTS}

The study was partly supported by the National Natural Science Foundation of China (Grant Number: 61571113 and Grant Number: 61671275), the Key Research and Development Programs of Jiangsu Province (Grant Number: BE2017735). The authors thank the support from the Southeast-Lenovo Wearable Heart-Sleep-Emotion Intelligent monitoring Lab.

\section{REFERENCES}

1. Vest A N., Li Q., Liu C Y., Nemati S., Shah A., Clifford G D.. Benchmarking heart rate variability toolboxes Journal of electrocardiology. 2017;50:744-747.

2. Salo M A., Huikuri H V., Seppanen T.. Ectopic beats in heart rate variability analysis: effects of editing on time and frequency domain measures Annals of Noninvasive Electrocardiology. 2001;6:5-17.

3. Nabil D., Reguig F B.. Ectopic beats detection and correction methods: A review Biomedical Signal Processing and Control. 2015;18:228244.

4. Berntson G G., Stowell J R.. ECG artifacts and heart period variability: don't miss a beat! Psychophysiology. 1998;35:127-132.

5. Singh B., Singh D., Jaryal A K., Deepak K K.. Ectopic beats in approximate entropy and sample entropy-based HRV assessment International Journal of Systems Science. 2012:43:884-893.

6. Clifford G D., Tarassenko L.. Quantifying errors in spectral estimates of HRV due to beat replacement and resampling IEEE transactions on Biomedical Engineering. 2005;52:630-638.

7. Tarkiainen T H., Kuusela T A., Tahvanainen K U O., et al. Comparison of methods for editing of ectopic beats in measurements of shortterm nonlinear heart rate dynamics Clinical Physiology and Functional Imaging. 2007;27:126-133.

8. Liu C Y., Li L P., Zhao L N., Zheng D C., Li P., Liu C C.. A combination method of improved impulse rejection filter and template matching for identification of anomalous intervals in RR sequences Journal of Medical and Biological Engineering. 2012;32:245-249.

9. Peltola M A.. Role of editing of RR intervals in the analysis of heart rate variability Frontiers in Physiology. 2012;3.

10. Goldberger A L., Amaral L A N., Glass L., et al. Physiobank, physiotoolkit, and physionet Circulation. 2000;101:e215-e220.

11. Zhao L N., Wei S S., Zhang C Q., et al. Determination of sample entropy and fuzzy measure entropy parameters for distinguishing congestive heart failure from normal sinus rhythm subjects Entropy. 2015;17:6270-6288.

12. Zhu K., Chemla D., Roisman G., et al. Overnight heart rate variability in patients with obstructive sleep apnoea: a time and frequency domain study Clinical and Experimental Pharmacology and Physiology. 2012;39:901-908.

Author: Chengyu Liu

Institute: Southeast University

Street: Sipailou 2\#

City: Nanjing

Country: China

Email: chengyu@seu.edu.cn 